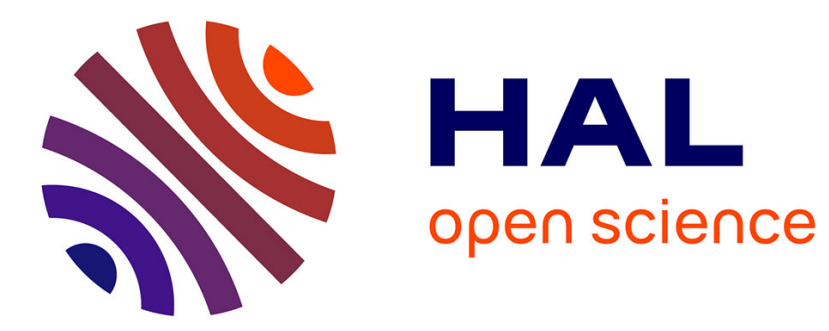

\title{
Wetting transitions of continuously varying or infinite order from a mean-field density-functional theory
}

Joseph Indekeu, Kenichiro Koga, Benjamin Widom

\section{To cite this version:}

Joseph Indekeu, Kenichiro Koga, Benjamin Widom. Wetting transitions of continuously varying or infinite order from a mean-field density-functional theory. Molecular Physics, 2011, 109 (07-10), pp.1297-1311. 10.1080/00268976.2011.556095 . hal-00692124

\section{HAL Id: hal-00692124 \\ https://hal.science/hal-00692124}

Submitted on 28 Apr 2012

HAL is a multi-disciplinary open access archive for the deposit and dissemination of scientific research documents, whether they are published or not. The documents may come from teaching and research institutions in France or abroad, or from public or private research centers.
L'archive ouverte pluridisciplinaire HAL, est destinée au dépôt et à la diffusion de documents scientifiques de niveau recherche, publiés ou non, émanant des établissements d'enseignement et de recherche français ou étrangers, des laboratoires publics ou privés. 


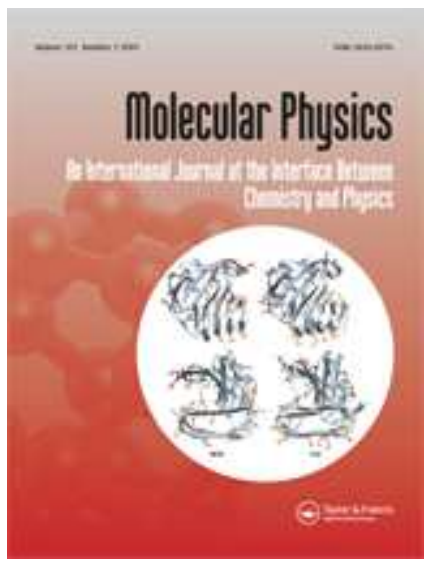

\section{Wetting transitions of continuously varying or infinite order from a mean-field density-functional theory}

\begin{tabular}{|c|c|}
\hline Journal: & Molecular Physics \\
\hline Manuscript ID: & TMPH-2010-0445.R1 \\
\hline Manuscript Type: & Special Issue paper - In honour of Bob Evans \\
\hline $\begin{array}{r}\text { Date Submitted by the } \\
\text { Author: }\end{array}$ & 29-Dec-2010 \\
\hline Complete List of Authors: & $\begin{array}{l}\text { Indekeu, Joseph; K.U.Leuven } \\
\text { Koga, Kenichiro; Okayama University } \\
\text { Widom, Benjamin; Cornell University }\end{array}$ \\
\hline Keywords: & wetting phase transition, density functional model \\
\hline
\end{tabular}

\section{SCHOLARONE Manuscripts}


The sensitivity of wetting phenomena and in particular (equilibrium) wetting phase transitions to details of the physico-chemical properties of substrate and adsorbate is remarkable and has been the subject of many investigations [1]. Recently it has been shown that the order of the wetting transition can be varied over an impressive range, from first to infinite order, as a result of small modifications in the parameters of a "minimal" mean-field density functional model for a three-phase equilibrium with two spatially varying densities [2]. While some aspects of these findings are reminiscent of earlier discoveries, such as the possibility of wetting transitions with continuously varying order and nonuniversal critical exponents in systems with a two-component order parameter [3], the appearance of infinite-order transitions in a standard model for wetting is unexpected. The present paper aims at a deeper understanding of these findings, especially through a more detailed analysis of the analytical calculations and approximations that quantitatively reproduce, or predict, and explain the main computational (numerical) results.

Consider three phases, $\alpha, \beta$ and $\gamma$, and their mutual interfaces, that meet along a line of common contact (Fig.1a). Alternatively, one of the three phases, say $\beta$, may intrude between the other two, and only two interfaces result, which do not intersect (Fig.1b). The first possibility is termed partial wetting (or incomplete wetting), or simply nonwet, and the second complete wetting, or simply wet. In the latter case, the phases are ordered along the $z$-direction, which is perpendicular to the interfaces. The transition between the two possibilities is termed the wetting phase transition and it arises when one of the dihedral contact angles in the configuration of Fig.1a, for example the angle between the $\alpha \beta$ and $\beta \gamma$ interfaces, becomes zero. The following triangle inequality between the interfacial tensions [4] is satisfied in all considered configurations,

$$
\sigma_{\alpha \gamma} \leq \sigma_{\alpha \beta}+\sigma_{\beta \gamma},
$$

the equality being reserved for complete wetting and the strict inequality for partial wetting. As usual, the spreading coefficient is defined through

$$
S=\sigma_{\alpha \gamma}-\left(\sigma_{\alpha \beta}+\sigma_{\beta \gamma}\right)
$$

In thermodynamic equilibrium, $S \leq 0$, in view of (1).

\footnotetext{
* Dedicated to Bob Evans on the occasion of his 65th birthday
} 
(a)

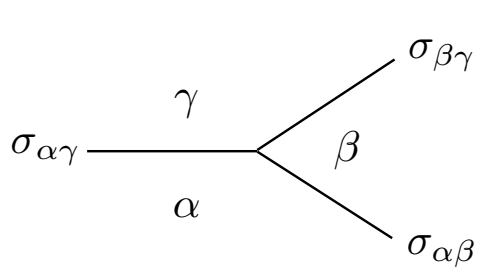

(b)

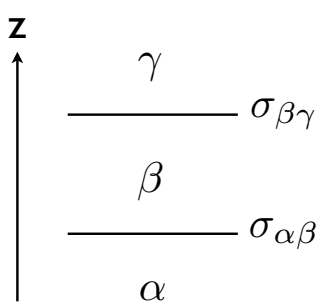

FIG. 1: Two possible configurations for three-phase equilibrium. (a) Partial wetting: the three phases $\alpha, \beta$ and $\gamma$ are in mutual contact. The three interfaces, with interfacial tensions $\sigma_{\alpha \beta}, \sigma_{\beta \gamma}$ and $\sigma_{\alpha \gamma}$ meet at a common contact line. (b) Complete wetting. Phase $\beta$, called the wetting phase, intrudes between phases $\alpha$ and $\gamma$.

Density-functional theories of interfacial structure and tension and of surface phase transitions (wetting transitions) have been brilliantly applied and reviewed by Bob Evans [5-7]. The mean-field density-functional model we study here is characterized by the following functional of two spatially varying densities $\rho_{1}(\mathbf{r})$ and $\rho_{2}(\mathbf{r})$, giving the excess free energy per unit area of an interface, oriented perpendicular to $z$,

$$
\hat{\sigma}\left[\rho_{1}, \rho_{2}\right]=\int_{-\infty}^{\infty} d z\left\langle\left\{\frac{1}{2}\left(\nabla \rho_{1}(\mathbf{r})\right)^{2}+\frac{1}{2}\left(\nabla \rho_{2}(\mathbf{r})\right)^{2}+F\left(\rho_{1}(\mathbf{r}), \rho_{2}(\mathbf{r}) ; a, b\right)\right\}\right\rangle_{\{x, y\}},
$$

where the outer brackets denote that the integrand is averaged over the directions $x$ and $y$ parallel to the interface and the free-energy density (per unit volume) $F$ is the following 6 -th order polynomial function

$$
F\left(\rho_{1}, \rho_{2} ; a, b\right)=\left(\left(\rho_{1}+1\right)^{2}+\rho_{2}^{2}\right)\left(\left(\rho_{1} / a\right)^{2}+\left(\rho_{2}-b\right)^{2}\right)\left(\left(\rho_{1}-1\right)^{2}+\rho_{2}^{2}\right)
$$

The model features (only) two parameters, $a$ and $b$. The latter, $b$, is a control parameter that allows wetting to be induced. Although, dimensionally, it stands on the same footing as the density $\rho_{2}$, it is physically more akin to a field variable through its (possible) dependence on temperature, chemical potentials, external magnetic or electric fields, or some combination of these. The former, $a$, is in general an asymmetry parameter, $a=1$ being its symmetric value. This asymmetry parameter can, in some systems, be related to spatial anisotropy. We will discuss concrete examples of asymmetry and anisotropy further on.

Clearly, for arbitrary $a$ and $b, F$ reaches its minimum value, equal to zero, when the densities reach their values appropriate to each of the bulk phases $\alpha, \beta$ and $\gamma$, at $z= \pm \infty$. These densities are

$$
\begin{aligned}
& \alpha \text { phase }: \rho_{1}=-1, \rho_{2}=0 \\
& \beta \text { phase }: \rho_{1}=0, \rho_{2}=b \\
& \gamma \text { phase }: \rho_{1}=1, \rho_{2}=0
\end{aligned}
$$

We treat our problem in mean-field theory and neglect the dependence of the densities on the directions $x$ and $y$ parallel to the interface. Then, the equilibrium density profiles $\rho_{1}(z), \rho_{2}(z)$ that minimize the functional (3) can be viewed, in a mechanical analogy [4], as least-action trajectories of a particle traveling in the two-dimensional $\left(\rho_{1}, \rho_{2}\right)$ space at time $t \equiv z$, on a triple-hill potential $V \equiv-F$. The "boundary conditions" or "initial" and "final" conditions correspond to the two bulk phases that are separated by the interface that is being considered. For example, the interfacial tension $\sigma_{\alpha \beta}$ is obtained by minimizing $\hat{\sigma}\left[\rho_{1}, \rho_{2}\right]$ subject to the boundary conditions that the bulk $\alpha$ phase is reached at $z=-\infty$ and the bulk $\beta$ phase at $z=\infty$.

We consider the wetting or nonwetting of the $\alpha \gamma$ interface by the $\beta$ phase. Fig. 2 shows typical equilibrium density profiles, corresponding to the $\alpha \beta$ interface (Fig.2, a and b), the nonwet $\alpha \gamma$ interface (Fig.2, c and d) and the wet $\alpha \gamma$ interface (Fig.2, e and $\mathrm{f}$ ).

It is instructive to study the interfacial trajectories in the $\left(\rho_{1}, \rho_{2}\right)$-plane. Fig.3 shows the trajectories for the nonwet and the wet $\alpha \gamma$ interface. Using this picture, it is possible to distinguish straightforwardly between a first-order and 


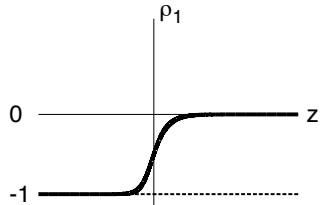

(a)
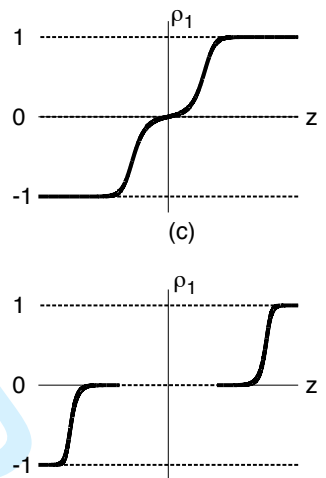

(e)

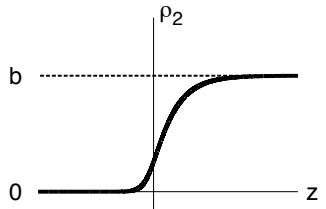

(b)

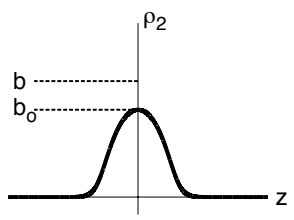

(d)

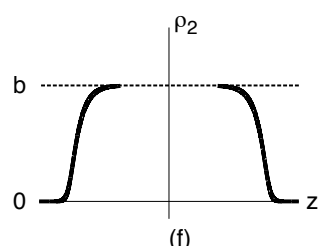

FIG. 2: Density profiles as a function of the spatial coordinate $z$. In (a) and (b) $\rho_{1}(z)$ and $\rho_{2}(z)$ are shown in the $\alpha \beta$ interface. In (c) and (d) $\rho_{1}(z)$ and $\rho_{2}(z)$ are shown in the $\alpha \gamma$ interface when it is not wet by the $\beta$ phase. In (d) $\rho_{2}$ reaches a maximum value $b_{0}$ that is less than its value $b$ in the bulk $\beta$ phase. In (e) and (f) $\rho_{1}(z)$ and $\rho_{2}(z)$ are shown in the $\alpha \gamma$ interface when it is wet by the $\beta$ phase. The dotted lines, bridging the gap between the solid lines, in (e) and (f), indicate the macroscopic (infinite) thickness of the wetting layer of $\beta$.

a continuous (higher-order) wetting transition. At a first-order wetting transition, the interfacial tensions $\sigma_{\alpha \gamma}$ for the nonwet (dashed) and for the wet (solid) trajectory are equal, while the trajectories do not coincide, i.e., $b_{0}<b$. Thus, the equilibrium structure of the $\alpha \gamma$ interface is discontinuous at first-order wetting, in the sense that the equilibrium density profiles change discontinuously from the "non-wet" to the "wet" profiles. In contrast, at a continuous wetting transition at bulk three-phase coexistence, for which we adopt the familiar term "critical wetting", the interfacial tensions for the wet and non-wet trajectories become equal only when the two trajectories coincide, asymptotically, in the limit $b_{0} \rightarrow b$.

This paper is organized as follows. In Section II we present an exploratory survey of various possible wetting transitions, classified according to their (increasing) order, and provide some concrete examples sampled from a diversity of systems. In Section III we turn to a detailed discussion of analytical approximations useful for extracting the asymptotic properties, close to wetting, of our density-functional model. In Section IV a somewhat simpler density-functional model is introduced, for which wetting transitions of infinite order are possible. The paper closes with conclusions and an outlook on a possible experimental system that might display infinite-order wetting.

\section{RECONNAISSANCE}

\section{A. First-order wetting}

The paradigm of a first-order wetting transition is that which was studied by Moldover and Cahn [8] and by Bonn and co-workers [9] in binary liquid mixtures "adsorbed" at their common vapor ( $\gamma$ phase). When a small amount of water is added to a mixture of methanol ( $\beta$ phase) and cyclohexane ( $\alpha$ phase), the liquid-vapor interface undergoes a qualitative change. At very low water concentration $x \ll 1$ a gravity-thinned [10] wetting layer of methanol is present at the cyclohexane-vapor interface (wet state), while a genuine three-phase contact is apparent at higher 


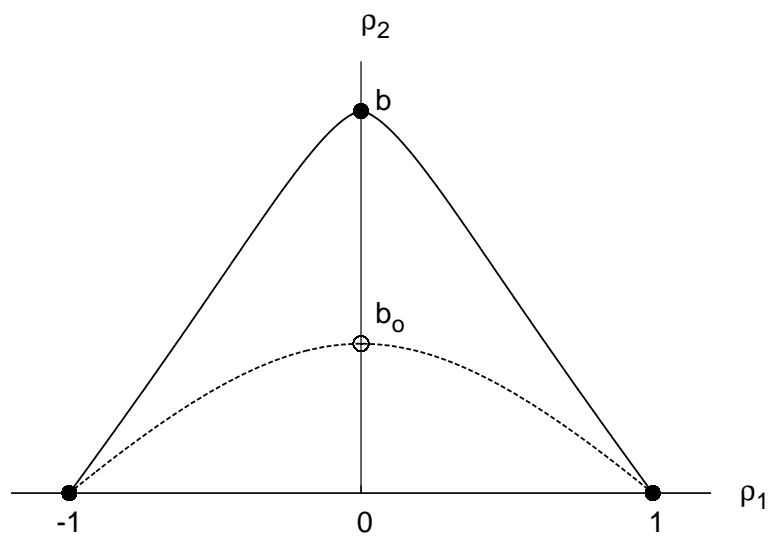

FIG. 3: Computed trajectories in the $\left(\rho_{1}, \rho_{2}\right)$-plane, obtained by eliminating $z$ from the density profiles $\rho_{1}(z)$ and $\rho_{2}(z)$. The model parameters are $a=1.6$ and $b=0.973$. They correspond to a first-order wetting transition. The solid line is a composite of the $\alpha \beta$ and $\beta \gamma$ interfaces and is therefore also the trajectory for the $\alpha \gamma$ interface when it is wet by $\beta$. The dashed curve is the trajectory for the $\alpha \gamma$ interface when it is not wet by $\beta$. The non-wet and wet trajectories are clearly distinct, but the corresponding interfacial structures have the same surface free energy.

water content (nonwet state). In this nonwet state the $\alpha \beta$ interface makes a finite contact angle $\theta$ with the (nearly horizontal) $\alpha \gamma$ interface. At the transition between the two regimes, at water concentration $x=x_{w}$, the spreading coefficient vanishes, with a discontinuity in its slope, as a function of $x$. We can characterize the singularity in $S$ at wetting by writing

$$
S=\sigma_{\alpha \beta}(\cos \theta-1) \propto\left|x-x_{w}\right|^{2-\alpha_{s}} ; \text { with } \alpha_{s}=1
$$

The critical exponent $\alpha_{s}$ is usually referred to as the "surface specific heat exponent" associated with the wetting transition. In principle, it can take any value less than or equal to 1 . Clearly, for first-order wetting, $\alpha_{s}=1$, since $S$ vanishes proportionally to the first power of the distance to the transition, measured in a suitable field variable (in this case water concentration).

In the density-functional model defined through (3) the wetting transition is found to be of first order for $a>1$. Its locus is given by a curve $b_{w}(a)$ or $a_{w}(b)$, depending on which parameter, $b$ or $a$, is used to control the distance to the transition. An example of interfacial trajectories at first-order wetting, computed in our model, is shown in Fig.3. It can be proven that a sufficient condition for the wetting transition to be of first order is $a>2$ [2]. In this regard we note that, in particular, for one of the mean-field density functional models studied earlier [11, 12] one can calculate the corresponding value of $a$ and verify that $a>2$ at the wetting transition. The transition was found to be of first order, indeed.

\section{B. Second-order wetting}

Probably the best known second-order wetting transition is the critical wetting transition of the two-dimensional Ising model discovered by Abraham [13]. For that phenomenon one finds, e.g., upon approach of the critical wetting temperature $T_{w}$,

$$
S=\sigma_{\alpha \beta}(\cos \theta-1) \propto\left|T-T_{w}\right|^{2-\alpha_{s}} ; \text { with } \alpha_{s}=0,
$$

implying a "parabolic" vanishing of the spreading coefficient at wetting (and a discontinuity in the surface specific heat, which is defined here through the second derivative of the spreading coefficient with respect to temperature). For any continuous wetting transition, in particular this second-order one, there is another critical exponent, $\beta_{s}$, which is independent of $\alpha_{s}$ and which describes the divergence of the equilibrium wetting layer thickness $\ell$ upon approach of the complete wetting state. One standardly writes

$$
\ell \propto\left|T-T_{w}\right|^{\beta_{s}} ; \text { with in this case } \beta_{s}=-1
$$


The exponent value $\beta_{s}=-1$ is not only found for critical wetting in the $2 \mathrm{~d}$ Ising model, for which thermal fluctuations are important, but also for critical wetting in 3d systems with long-range forces (with algebraic decay), for which thermal fluctuations are unimportant (mean-field theory applies) [14]. In contrast, if we study second-order wetting $\left(\alpha_{s}=0\right)$ in systems with short-range forces, then, typically the wetting layer thickness diverges more weakly (logarithmically),

$$
\ell \propto \log \left(\left|T-T_{w}\right|^{-1}\right) ; \text { so that } \beta_{s}=0(\log )
$$

However, it was quite a surprise when, in 1999, in binary liquid mixtures of nonane and methanol, mean-field and short-range critical wetting signatures were observed experimentally, i.e., $\alpha_{s}=0$ and $\beta_{s}=0(\log )$ [15]. Apparently, the critical wetting transitions in those systems occur so close to the bulk critical point that effects of long-range (van der Waals) forces are negligible. Furthermore, thermal fluctuation effects on short-range critical wetting were not observed. This is consistent with the current insight that these effects are expected to show up only extremely close to the transition point [18].

In the context of our mean-field density-functional model, in which long-range forces are fully ignored, a (shortrange) second-order wetting transition was found for $a=1$ (symmetric model), when $b$ is lowered towards $b_{w}=0.681 \ldots$ [16]. For that transition, $2-\alpha_{s}=2$, whence the qualification "second-order wetting", and we verified numerically that $\beta_{s}=0(\log )$, as expected.

\section{Third-order wetting}

It may seem odd that third-order wetting transitions are the subject of a separate subsection in our Reconnaissance, but it is fully justified, not only because transitions of higher than second order are found in our density-functional model for $a<1$, as we will discuss shortly, but also because, remarkably, third-order wetting transitions were predicted to occur in density functional models for systems with long-range (van der Waals) forces, and they have been observed experimentally in liquid mixtures (e.g., pentane on water) [17]. For that system, with phases $\alpha$ (water), $\beta$ (pentane liquid) and $\gamma$ (moist pentane vapor),

$$
S=\sigma_{\beta \gamma}(\cos \theta-1) \propto\left|T-T_{w}\right|^{2-\alpha_{s}} ; \text { with } \alpha_{s}=-1,
$$

so that $2-\alpha_{s}=3$, which is characteristic for critical wetting in systems with non-retarded van der Waals forces, and

$$
\ell \propto\left|T-T_{w}\right|^{\beta_{s}} ; \text { with } \beta_{s}=-1
$$

The latter result is quite generally a consequence of the algebraic decay of the intermolecular forces.

Within our mean-field density-functional model, a third-order wetting transition does not arise in an isolated manner but rather as a non-special member of a family of critical wetting transitions of continuously varying order, as will be discussed next. For these transitions the exponent $\alpha_{s}$ varies as a function of the asymmetry parameter $a$ and the divergence of the wetting layer thickness is found to be logarithmic $\left(\beta_{s}=0(\log )\right)$.

\section{Non-universal wetting, with continuously varying order}

Since the pioneering work of Hauge it is well known that inhomogeneous systems in which two different length scales occur, can display non-universal critical wetting phenomena [3]. These two different lengths can pertain to decay lengths associated with two distinct order parameters or order parameter components. This is the case we will be interested in here. Alternatively, even in a single order parameter theory non-universal wetting can arise when the decay length of the "wall potential" varies relative to the bulk correlation length [19].

Before turning to the manifestation of non-universal wetting in our density-functional model, we discuss briefly two concrete physical systems for which non-universal critical wetting exponents have been identified. The first of these is a two-component magnetic vector model with cubic anisotropy [20] and the second is a type-I superconductor with surface enhancement of superconductivity [21].

\section{Ferromagnet with cubic anisotropy}

The order parameters in this anisotropic vector model of a semi-infinite ferromagnet are the components $M_{x}$ and $M_{y}$ of a magnetization function $\mathbf{M}(z)$. They play roles similar to those of our densities $\rho_{1}$ and $\rho_{2}$. Particularly 
relevant for us is to examine how the anisotropy is defined through parameters entering the free-energy density $F(\mathbf{M})$, which in zero external magnetic field is taken to be of the form

$$
F(\mathbf{M} ; \epsilon)=\frac{t}{2} \mathbf{M} \cdot \mathbf{M}+\frac{1}{4}(\mathbf{M} \cdot \mathbf{M})^{2}+\epsilon M_{x}^{2} M_{y}^{2},
$$

with $t \equiv\left(T-T_{c}\right) / T_{c}(<0)$ the reduced temperature and $T_{c}$ the Curie temperature.

In the absence of crystal lattice anisotropy the isotropic form, with $\epsilon=0$, applies. In the presence of cubic anisotropy the magnetization preferentially aligns along a crystal axis, $x$ or $y$, for $\epsilon>0$, and along a body diagonal for $\epsilon<0$. We now examine how the anisotropy parameter $\epsilon$ can be related to our asymmetry variable $a$ introduced in (4). From now on we focus on the regime $\epsilon>0$. In this regime, four bulk phases, with magnetizations $\mathbf{M}=( \pm \sqrt{-t}, 0)$ and $\mathbf{M}=(0, \pm \sqrt{-t})$, coexist for $t<0$ and zero bulk field (see Fig.1 in [20]). We reconsider the example discussed in [20] and assume that, without loss of generality, the "A" phase with bulk order parameter $\mathbf{M}_{A}=(\sqrt{-t}, 0)$ is the wetting phase. To define the relevant length scales it suffices to expand the free energy density (12) to quadratic order about its minimum at $\mathbf{M}_{A}$. In terms of the "potential energy" $V(\mathbf{M}) \equiv F\left(\mathbf{M}_{A}\right)-F(\mathbf{M})$ this gives

$$
V(\mathbf{M} ; \epsilon) \approx-\frac{1}{2}\left(\left(\frac{\xi_{0}}{\xi_{x}(\epsilon)}\right)^{2}\left(M_{x}-\sqrt{-t}\right)^{2}+\left(\frac{\xi_{0}}{\xi_{y}(\epsilon)}\right)^{2} M_{y}^{2}\right),
$$

where $\xi_{0}$ is a constant reference length and $\xi_{i}$ a length related to the (inverse) curvature of the potential along the $i$-axis in the order parameter plane, which sets the decay length for the $i$-component of the order parameter towards its value in bulk. Consistently with (4) we now define the asymmetry parameter $a$ through

$$
\frac{1}{a} \equiv \frac{\xi_{x}}{\xi_{y}}=\sqrt{\frac{\epsilon}{2}} \equiv \sqrt{\frac{\lambda-1}{2}}
$$

where we defined $\lambda \equiv 1+\epsilon$ to allow easy comparison with the anisotropy variable, $\lambda-1$, used in [20]. Clearly, the "symmetric" case for the wetting problem corresponds to $a=1$, or $\lambda=3(\epsilon=2)$. Note that this is different from the value $\lambda=1(\epsilon=0)$, for which the bulk free energy density is isotropic in $\mathbf{M}$.

For $a>1$ first-order wetting was predicted, while for $a<1$ critical wetting results, with the standard (universal) second-order wetting exponent $\alpha_{s}=0$ for $0<a \leq 1 / 2$ and a non-universal exponent $\alpha_{s}=(1-2 a) /(1-a)$ or $2-\alpha_{s}=1 /(1-a)$ for $1 / 2<a<1$ [20]. Note that the order of the transition varies continuously between 2 (the universal value valid for $a \leq 1 / 2$ ) and arbitrarily high order (for $a \rightarrow 1$ ). However, for the symmetric case $a=1$ a standard second-order transition $\left(\alpha_{s}=0\right)$ was established [22]. In all critical wetting regimes $(0<a \leq 1)$, a logarithmic divergence of the wetting layer thickness was reported $\left(\beta_{s}=0(\log )\right)$.

\section{Type-I superconductors with surface enhancement}

The two order parameters in this case are the complex superconducting wave function $\psi$ and the magnetic vector potential A. In a suitable semi-infinite geometry the Meissner state and the normal state of a type-I superconductor can be described by the scaled real (and positive) superconducting order parameter $\phi(z)$ and a scaled vector potential component $\mathcal{A}(z)$, where $z$ is the distance from the surface into the material. The wetting transition in this material (in which the Meissner phase intrudes between the surface and the normal phase) has been studied within GinzburgLandau theory [21] and verified experimentally for Sn [23]. A necessary condition for this transition to occur is the presence of surface enhancement of superconductivity, which can be induced by polishing or ion bombardment of the surface [23]. The "asymmetry" in this system is fundamental and arises from the qualitative difference between the superconducting coherence length $\xi$ and the magnetic penetration depth $\lambda$. The ratio $\kappa=\lambda / \xi$ is a material constant (near the bulk critical temperature $T_{c}$ ). In order to see how the asymmetry parameter $a$ can be defined, we examine the expansion of the bulk free energy density about the order parameter values in the bulk superconducting phase, $\phi=1 / \kappa$ and $\mathcal{A}=0$, where we follow the scaling of the variables as employed in the second paper in [21]. The potential energy is given by

$$
V(\phi, \mathcal{A} ; \kappa)=\frac{1}{2} \phi^{2}-\frac{1}{2} \mathcal{A}^{2} \phi^{2}-\frac{1}{4} \kappa^{2} \phi^{4}-\frac{1}{4 \kappa^{2}}
$$

Expanding, to second order, about the bulk superconducting phase point, leads to

$$
V(\phi, \mathcal{A} ; \kappa) \approx-\left(\phi-\frac{1}{\kappa}\right)^{2}-\frac{\mathcal{A}^{2}}{2 \kappa^{2}}
$$


Comparison with (4) allows us to define the asymmetry parameter as

$$
a=\sqrt{2} \kappa
$$

Guided by the seminal insight of Hauge [3] concerning the possible dependence of critical wetting singularities on the ratio of two competing lengths, the critical wetting transition in type-I superconductors [21] was scrutinized along similar lines of reasoning [24]. Mathematically, a branch with universal second-order wetting exponents and a branch with non-universal exponents that vary continuously with $\kappa$ are possible. Specifically, for $a<1$ critical wetting is possible with the standard (universal) second-order wetting exponent $\alpha_{s}=0$ for $0<a \leq 1 / 2$ and with a non-universal exponent $\alpha_{s}=(1-2 a) /(1-a)$, or $2-\alpha_{s}=1 /(1-a)$ for $1 / 2<a<1$, corresponding to critical wetting transitions of arbitrarily high order. However, accurate model computations have shown that a first-order wetting transition takes place for $0<\kappa<0.374$ [21], which corresponds to $0<a<0.529$. Consequently, only the non-universal critical wetting transition is physically relevant in this system. Further, a logarithmic divergence of the wetting layer thickness at critical wetting was established $\left(\beta_{s}=0(\log )\right)[21]$.

The symmetric point in this model, $a=1$, or $\kappa=1 / \sqrt{2}$, corresponds to the bulk multicritical transition between type-I and type-II superconductivity. Consequently, for $a \geq 1$ a discussion of wetting transitions is meaningless since a stable interface between Meissner and normal phases does not exist. Notwithstanding the presence of this physical "frontier" at $a=1$, the wetting layer thickness displays an interesting divergence when, coming from a partial wetting, the limit $a \rightarrow 1$ is considered [24-27]. In particular, $\ell \propto(1-a)^{-1}$, in this limit, but a macroscopic wetting layer is thermodynamically unstable for $a=1$. Therefore, in this physical system this divergence is not associated with a wetting transition.

After this overview of the non-universal continuous wetting transition in these two concrete systems, we return to our density-functional model described by (3). In this model a non-universal critical wetting transition is found for $a<1$, upon lowering $b$ towards $b_{w}=0$ [2]. For this transition, $\alpha_{s}=(1-2 a) /(1-a)$, or $2-\alpha_{s}=1 /(1-a)$. Note that the order of the transition varies continuously and increases to an arbitrarily high value for $a \rightarrow 1$. It is furthermore interesting to note that, in contrast with expectations based on previous models and scaling arguments $[3,20,21,24]$, also for $a<1 / 2$, and at least down to values of $a \approx 0.2$, non-universal behavior is found instead of the universal second-order transition. Further, for $a<1$, our computations confirm a logarithmic divergence of the wetting layer thickness $\left(\beta_{s}=0(\log )\right)$ for $b \rightarrow b_{w}=0$, in line with the predictions of the analytical approximations outlined in Section III.

\section{E. Infinite-order wetting}

Infinite-order wetting transitions have hitherto only been encountered under rather special circumstances. For example, a subregime of the intermediate (thermal) fluctuation regime for wetting transitions in two dimensions consists of infinite-order transitions [28]. It was therefore quite a surprise that they show up in a mean-field density functional model with a multi-component order parameter [2]. Previous studies of similar models [3, 20] missed them, presumably because they do not occur when the obvious control parameter of wetting is varied, but instead, they occur upon varying the asymmetry parameter $a$, related to a ratio of length scales associated with the decay of the order parameter components. The obvious point of view is to study wetting at arbitrary but fixed value of $a$. Ironically, in the case of type-I superconductors an infinite-order transition was the first to be uncovered while studying wetting [25], but it is not a wetting transition [21, 24, 27].

In our density-functional model a segment of infinite-order critical wetting transitions is found for $a=1$ (symmetric case) and for $0<b<b_{w}(1)=0.681 \ldots$ [2]. This $b_{w}(1)$ is defined as the value of $b$ at the second-order wetting transition found for $a=1$. The singularity in the spreading coefficient near wetting is predicted, based on analytic arguments, to be of the form

$$
-S \propto\left(b / b_{w}(1)\right)^{2 a /(1-a)}
$$

This can be cast in a form which more conspicuously brings out the exponential singularity arising in the limit $a \uparrow 1$,

$$
-S \propto e^{-C /(1-a)} ; \text { with } C>0
$$

This has been confirmed numerically. Interestingly as well, we verified numerically and by analytical arguments that the wetting layer thickness diverges in the algebraic manner, for $a \uparrow 1$,

$$
\ell \propto(1-a)^{-1}
$$

implying $\beta_{s}=-1$. This represents a much stronger divergence than the logarithmic law found in the other regimes discussed in this paper. 


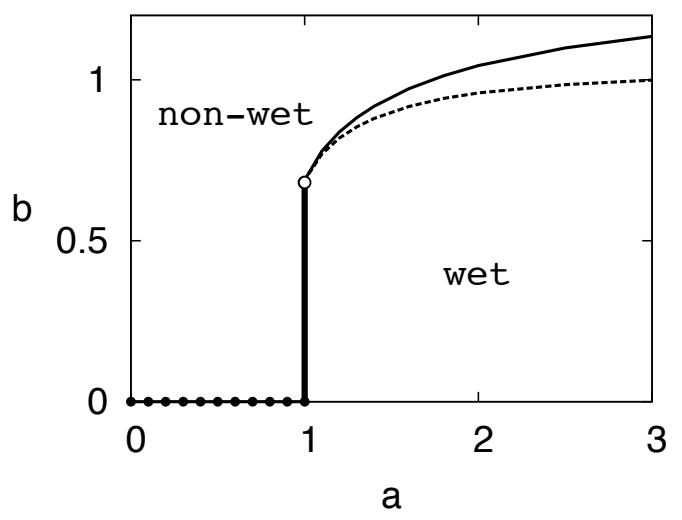

FIG. 4: Computed global wetting phase diagram in the $(a, b)$-plane. The phase boundary between wet and non-wet states consists of a segment of first-order wetting $\left(a>1\right.$; solid line), a second-order wetting point $\left(a=1, b=b_{w}(1) \approx 0.681\right)$, a segment of infinite-order wetting $(a=1$; vertical line), and a segment of wetting transitions of continuously varying order $\left(a<1, b=b_{w}=0\right.$; line decorated with dots). The thin dashed line $a>1$ is the spinodal of the non-wet states in the wet regime.

Figure 4 presents the global wetting phase diagram of our model in the $(a, b)$-plane. The segments of first-order (thin line; numerically computed), infinite-order (thick line at $a=1$ ) and continuously-varying-order wetting transitions (line with dots at $b=0$ ) are shown as solid curves, together with the isolated second-order transition point at $a=1$. In states above and to the left of the wetting phase boundary the $\alpha \gamma$ interface is not wet by $\beta$, while in the complementary sector it is wet by $\beta$. The dashed curve in the figure for $a>1$ is the numerically determined limit of metastability of the non-wet states in the region where the equilibrium configuration is that of complete wetting. At points $(a, b)$ in the region below the dashed line we no longer find a solution to the Euler-Lagrange equations for a non-wetting trajectory such as in Figs.2c-d or the dashed curve in Fig.3.

\section{ANALYTICAL APPROXIMATIONS TO THE MODEL}

Here we study our model from the point of view of applying a constraint on the order parameter profiles, and subsequently minimizing an effective free energy function with respect to the constraint. Due to the approximation of restricting the order parameter profiles to a judiciously chosen subset of all suitable functions, our approach is not exact for our model but may still capture the correct singular behavior of the spreading coefficient $S$ and the layer thickness $\ell$ near a continuous wetting transition $(a \leq 1)$. Therefore, the predictions of this analytical approximation must be checked against the results of "numerically exact" model computations.

\section{A. Interface potential approach}

In this approach we follow closely the lines of reasoning of, and experience gathered in, previous works on twocomponent order parameters [3, 20,24]. Two main assumptions enter: i) an accurate description of the trajectories in the $\left(\rho_{1}, \rho_{2}\right)$-plane in the vicinity of the $\beta$-phase point is essential for capturing the correct thermodynamic singularities at critical wetting; and ii) a non-wet state can be parameterized by a single "collective coordinate", $\ell$, which "measures" the thickness of the wetting layer and serves as an effective scalar order parameter for wetting.

Assumption i) prompts us to expand (4) to second order about $(0, b)$, which provides the approximation

$$
F\left(\rho_{1}, \rho_{2} ; a, b\right) \approx F^{(2)}\left(\rho_{1}, \rho_{2} ; a, b\right) \equiv \frac{A}{2}\left(\left(\rho_{1} / a\right)^{2}+\left(\rho_{2}-b\right)^{2}\right)
$$


with $A=2\left(1+b^{2}\right)^{2}$ a (finite) prefactor. Solving the Euler-Lagrange equations

$$
\partial F^{(2)} / \partial \rho_{1}=d^{2} \rho_{1} / d z^{2}, \quad \partial F^{(2)} / \partial \rho_{2}=d^{2} \rho_{2} / d z^{2}
$$

within this "harmonic" approximation, leads to the (general) solutions, expressed in the scaled distance $Z \equiv \sqrt{A} z$,

$$
\begin{aligned}
& \rho_{1}(Z)=a_{1} e^{Z / a}+b_{1} e^{-Z / a} \\
& \rho_{2}(Z)=b+a_{2} e^{Z}+b_{2} e^{-Z}
\end{aligned}
$$

and we define $b_{0}^{(2)} \equiv \rho_{2}(0)$, where the superscript refers to the harmonic approximation. Symmetry considerations lead us to fix the position $z=0$ to the "middle" of the layer of $\beta$ and to restrict our attention to finding solutions for $z \in[0, \infty)$ with the boundary conditions, or "initial conditions" at $z=0$,

$$
\begin{aligned}
\rho_{1}(0) & =0 \\
d \rho_{2}(Z) /\left.d Z\right|_{0} & =0
\end{aligned}
$$

These imply $b_{1}=-a_{1}$ and $b_{2}=a_{2}$. This still leaves two parameters undetermined. We cannot (yet) impose the "final conditions" for $z \rightarrow \infty$ because our approximation $F^{(2)}$ to $F$ is not useful in this limit. However, following [3] we can eliminate one more parameter by requiring that the first integral of the Euler-Lagrange equation, $E$, be a "constant of the motion" along the entire trajectory. This constant can be determined, e.g., in the bulk $\gamma$ phase, where the $z$-derivatives of the densities as well as the function $F$ attain the value zero,

$$
E=\frac{1}{2} \dot{\rho}_{1}^{2}+\frac{1}{2} \dot{\rho}_{2}^{2}-F=0
$$

Applying this at $z=0$, where our approximation $F^{(2)}$ to $F$ is accurate (provided $b_{0}^{(2)}$ is sufficiently close to $b$ ) implies $\left(a_{1} / a\right)^{2}=a_{2}^{2}$. Thus we have $a_{2}=-a_{1} / a$ since $b_{0}^{(2)}-b=2 a_{2}<0$ and $a_{1}>0$ (because $\left.\dot{\rho}_{1}(0)>0\right)$. Further, note that $\left(\rho_{2}-b_{0}^{(2)}\right) / \rho_{1} \sim-Z / 2$ for small $z$, so that the trajectory starts parallel to the $\rho_{1}$-axis, from the point $\left(0, b_{0}^{(2)}\right)$, in the $\left(\rho_{1}, \rho_{2}\right)$-plane.

Provided $a_{1}$ is chosen sufficiently small, the approximate (non-wet) trajectory that we are now constructing, intersects, at a position denoted by $z=z_{x}$, the exact wet trajectory associated with the interface between the $\beta$ and $\gamma$ phases, in the $\left(\rho_{1}, \rho_{2}\right)$-plane [29]. We now define a basically new model by imposing that, for $z>z_{\times}$, from the point of intersection up till the $\gamma$ phase point at $(1,0)$ our approximate trajectory be identical to the exact wet trajectory in the $\left(\rho_{1}, \rho_{2}\right)$-plane. Fig.5 illustrates our strategy. It is important to stress that, in the original model, the non-wet trajectory does not intersect the exact wet trajectory before both meet at the $\gamma$ phase point (see Figs.3 or 5 ). The intersection we consider is thus entirely an artifact of the different starting point (at $\rho_{2}(0)$ somewhere between $b$ and the exact $b_{0}$ ) of the non-wet trajectory. The intersection is a property of the new model, not present in the original model.

The interfacial trajectory, denoted by $\rho_{0}$, takes the following form in the vicinity of the $\beta$ phase,

$$
\begin{aligned}
& \rho_{0,1}\left(Z^{\prime}\right)=a_{0,1} e^{Z^{\prime} / a} ; \text { with } a_{0,1}>0 \\
& \rho_{0,2}\left(Z^{\prime}\right)=b+a_{0,2} e^{Z^{\prime}} ; \text { with } a_{0,2}<0,
\end{aligned}
$$

with the convention that $z^{\prime}=-\infty$ in the $\beta$ phase and $z^{\prime}=0$ corresponds to the "middle" of the $\beta \gamma$ interface. Assuming that $z_{\times}$remains finite when the wetting transition is approached - an assumption which will be checked on its self-consistency further on - we define the scaled wetting layer thickness $L \equiv \sqrt{A} \ell$ as the scaled distance from the point $z=z_{\times}$to the point $z^{\prime}=0$. This then defines how the scaled distances $Z^{\prime}$ and $Z$ are related to one another,

$$
Z^{\prime}=Z-\left(Z_{\times}+L\right)
$$

so that, in particular, $Z_{\times}^{\prime}=-L$. Further, we will soon establish how $L$ depends on $Z_{\times}$.

Thus, at $z=z_{\times}$we match our trajectory with the wet solution and for $z>z_{\times}$we follow the latter up till the $\gamma$ phase. The matching conditions at $z=z_{\times}$read,

$$
\begin{aligned}
& \rho_{1}\left(Z_{\times}\right)=2 a_{1} \sinh \left(Z_{\times} / a\right)=\rho_{0,1}\left(Z_{\times}^{\prime}\right)=a_{0,1} e^{-L / a} \\
& \rho_{2}\left(Z_{\times}\right)=b-2\left(a_{1} / a\right) \cosh Z_{\times}=\rho_{0,2}\left(Z_{\times}^{\prime}\right)=b+a_{0,2} e^{-L}
\end{aligned}
$$




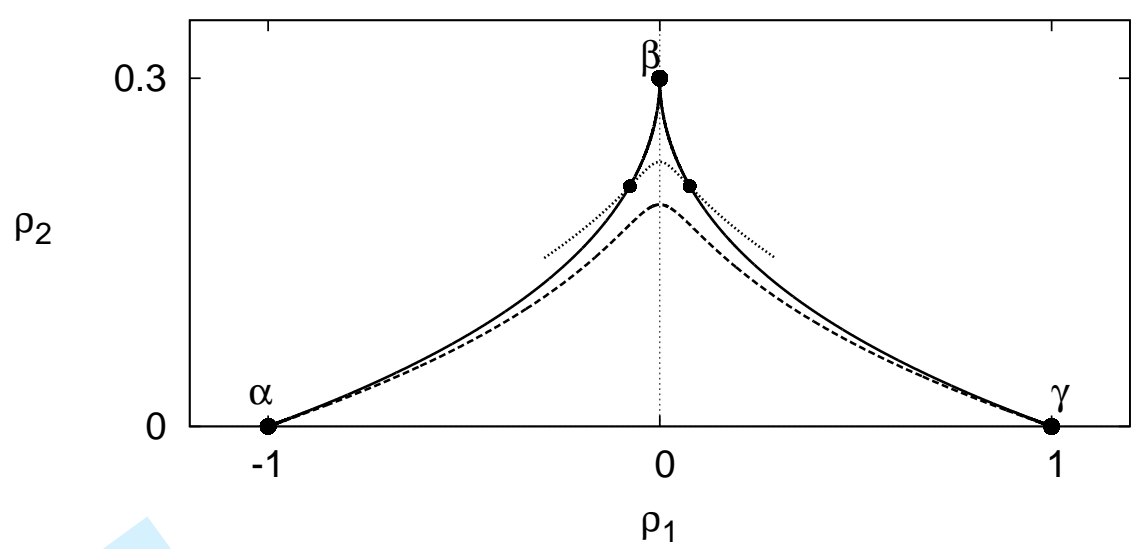

FIG. 5: Computed trajectories in the $\left(\rho_{1}, \rho_{2}\right)$-plane for $a=1 / 2$, obtained by eliminating $z$ from the density profiles $\rho_{1}(z)$ and $\rho_{2}(z)$. The solid line, from phase point $\alpha$ to $\gamma$ through $\beta$, is a composite of the $\alpha \beta$ and $\beta \gamma$ interfaces and is therefore also the exact trajectory for the $\alpha \gamma$ interface when it is wet by $\beta$. Note that, because $a<1$, this trajectory forms a cusp near $\beta$, in contrast with typical wet trajectories for $a>1$, which are flat near $\beta$ (Fig.3). The (lower) dashed curve is the exact trajectory for the $\alpha \gamma$ interface when it is not wet by $\beta$. The (upper) thin dashed curve is one of many possible non-wet trajectories in the harmonic approximation to the potential, which can be used only in the neighborhood of the $\beta$ phase point. It crosses the solid line in two points (black dots). The approximate non-wet trajectory, on which our analytic arguments are built, is - by definition - composed of the thin dashed curve, in between the crossings, and the solid line, on either side of the intersections. In between the crossings, the approximate non-wet trajectory is based on $\rho_{1}(Z)=2 a_{1} \sinh 2 Z$ and $\rho_{2}(Z)=b-4 a_{1} \cosh Z$, with, for illustrative purposes in this Figure, $b=0.3$ and $a_{1}=b^{2} / 5=0.018$; cf. [29].

From suitable combinations of these two conditions it follows how $L$ depends on $Z_{\times}$and vice versa, or, how $a_{1}$ depends on $Z_{\times}$and vice versa. For example, to illustrate the former we can write

$$
e^{-L\left(\frac{1}{a}-1\right)}=a \frac{\left|a_{0,2}\right|}{a_{0,1}} \frac{\sinh \left(Z_{\times} / a\right)}{\cosh Z_{\times}}
$$

which shows that, for fixed $a$ and $b$, besides the (fixed) parameters associated with the wet trajectory, only the "crossing" distance $Z_{\times}$controls the wetting layer thickness $L$ associated with our one-parameter family of approximations to the non-wet trajectory. It is therefore useful to think of $L$ as the (only) free parameter left in our approach. It is important to keep in mind that (29) is an approximate relationship, valid only to leading order, for large $L$. Only in that limit it is sufficient to work with just the leading terms, for $Z^{\prime} \rightarrow-\infty$, given in (26).

The surface free energy cost of the approximate non-wet trajectory on the interval $z \in\left[0, z_{\times}\right]$is now found using (3), (21), (23) and (28), and reads

$$
\hat{\sigma}_{\left[0, z_{\times}\right]}^{(2)}=\int_{0}^{z \times} d z\left\{\frac{1}{2} \dot{\rho}_{1}^{2}+\frac{1}{2} \dot{\rho}_{2}^{2}+F^{(2)}\left(\rho_{1}, \rho_{2}\right)\right\}=\frac{\sqrt{A}}{2}\left(a_{0,2}^{2} \tanh Z_{\times} e^{-2 L}+\frac{a_{0,1}^{2}}{a} \operatorname{coth} \frac{Z_{\times}}{a} e^{-2 L / a}\right),
$$

while on the interval $z \in\left[z_{\times}, \infty\right]$ the surface free energy cost of the approximate non-wet trajectory can be denoted by $\sigma_{\times \gamma} \equiv \hat{\sigma}_{\left[z_{x}, \infty\right]}$ and corresponds to a large fraction of the equilibrium interfacial tension $\sigma_{\beta \gamma}$. We need not compute this fraction, because, as can be seen by inspecting (2), it contributes equally to the surface free energies of both the approximate non-wet state and the exact wet state so that it gets canceled out in the spreading coefficient $S$.

Therefore, what remains to be calculated is the harmonic approximation [30] to the remaining fraction, $\sigma_{\beta \times}$, of the equilibrium interfacial tension $\sigma_{\beta \gamma} \equiv \sigma_{\beta \times}+\sigma_{\times \gamma}$. This is achieved through

$$
\sigma_{\beta \times}^{(2)}=\int_{-\infty}^{-\ell} d z^{\prime}\left\{\frac{1}{2} \dot{\rho}_{0,1}^{2}+\frac{1}{2} \dot{\rho}_{0,2}^{2}+F^{(2)}\left(\rho_{0,1}, \rho_{0,2}\right)\right\}=\frac{\sqrt{A}}{2}\left(a_{0,2}^{2} e^{-2 L}+\frac{a_{0,1}^{2}}{a} e^{-2 L / a}\right)
$$

In sum, we obtain the following harmonic approximation to the spreading coefficient $S$ associated with our oneparameter family of non-wet states for given $a$ and $b$,

$$
S^{(2)}(L)=2\left(\hat{\sigma}_{\left[0, z_{\times}\right]}^{(2)}-\sigma_{\beta \times}^{(2)}\right)=\sqrt{A}\left(a_{0,2}^{2}\left(\tanh Z_{\times}(L)-1\right) e^{-2 L}+\frac{a_{0,1}^{2}}{a}\left(\operatorname{coth} \frac{Z_{\times}(L)}{a}-1\right) e^{-2 L / a}\right)
$$


This expression can be interpreted as a "constrained" surface free energy excess of the non-wet state relative to the wet state, the constraint being a fixed, or imposed, value of the wetting layer thickness $\ell$. This constrained surface free energy difference is the so-called interface potential. Once more, we emphasize that this harmonic approximation is only valid to leading order(s) in $\exp (-2 L)$ and $\exp (-2 L / a)$. Corrections terms, not calculated in our approach, should be expected. Presumably these corrections are of order $\exp (-4 L)$ and $\exp (-4 L / a)$. Whether such possible corrections are relevant must be assessed case by case.

We may now attempt to obtain the harmonic approximation to the equilibrium spreading coefficient by minimizing this interface potential $V(\ell) \equiv S^{(2)}(L)$ with respect to $\ell$. Note that in view of (32) we have $V(\infty)=0$. The equilibrium wetting layer thickness $\hat{\ell}$ follows from

$$
\left.\frac{d V(\ell)}{d \ell}\right|_{\hat{\ell}}=0
$$

To proceed analytically is in general a nontrivial task since, as we have made explicit in (32), the crossing distance $Z_{\times}$ depends on $L$. Note that, for $a>1$, the second term in (32) is the leading one and its prefactor is positive. Therefore, for $a>1$ this approximation predicts the existence of a barrier in the interface potential and a (local) minimum at $L=\infty$. This implies a first-order wetting transition, consistently with our numerical computations for $a>1$. On the other hand, for $a<1$, the first term leads in (32) and it is negative, while the second term is positive. In this case the interface potential allows us to describe critical wetting in the limit $\left|a_{0,2}\right| \rightarrow 0$, as we will now show in more detail.

\section{B. Non-universal wetting, with continuously varying order: The case $a=1 / 2$}

It is instructive to explore first the special case $a=1 / 2$, for which (29) greatly simplifies to

$$
e^{-L}=\frac{\left|a_{0,2}\right|}{a_{0,1}} \sinh Z_{\times},
$$

to leading order in $\exp (-L)<<1$. Using this, we can eliminate $Z_{\times}$in favor of $L, a_{0,1}$ and $a_{0,2}$ in the interface potential. Subsequently, we calculate the leading terms, for large $\ell$, in the expansion of $V(\ell)$ in powers of $\exp (-L)$. This leads to the result

$$
V(\ell) / \sqrt{A}=-a_{0,2}^{2} e^{-2 L}-2 a_{0,1} a_{0,2} e^{-3 L}+\mathcal{O}\left(e^{-4 L}\right),
$$

and we recall $a_{0,2}<0$. We do not know the amplitude of the correction term, of order $\exp (-4 L)$, because it may contain a contribution from an "anharmonic" correction to (32). Nevertheless, using only the two leading terms it is possible to derive an approximate equilibrium wetting layer thickness using (33). We find

$$
e^{-\hat{L}}=\frac{\left|a_{0,2}\right|}{3 a_{0,1}}
$$

Note that, within this approximation, $z_{\times}(\hat{\ell})$ is finite since $\sinh Z_{\times}(\hat{L})=1 / 3$ for $a=1 / 2$, independently of $b$. The precise value of this constant $(1 / 3)$ is unimportant, as it does not affect the form of the critical singularities at wetting. Important is that our approximation is self-consistent because for finite $z_{\times}$the intersection point of the trajectories lies close to the $\beta$ phase point where our harmonic approximation is justified. Making the observation that $\left|a_{0,2}\right|=\mathcal{O}(b)$, in view of the second of Eqs.(26), we obtain a logarithmic divergence of the wetting layer thickness

$$
\hat{\ell} \propto \ln (1 / b)
$$

implying a critical wetting transition in the limit $b \rightarrow 0$. Besides the exponent $\beta_{s}=0(\log )$ we can also obtain the critical exponent of the surface free energy singularity, after inserting the equilibrium expression (36) into (either of) the two terms in (35). We obtain

$$
-S \propto b^{4}
$$

In order to obtain $\alpha_{s}$ explicitly we now generalize our calculations to $a \neq 1 / 2$. 


\section{Non-universal wetting, with continuously varying order: The more general case $a<1$}

For $a \neq 1 / 2$ we assume that $z_{\times}(\hat{\ell})$ is also a finite constant, as our inspection of the case $a=1 / 2$ suggested. The outcome of the calculations, in as far as the critical exponents at wetting are concerned, are quite robust to changing the value of this constant and to the approximations made to derive it. In the following we will accordingly simplify the algebra considerably by assuming that $z_{\times}$and even $z_{\times} / a$ are smaller than unity in such a way that the hyperbolic functions can be safely approximated by their expansions to first order in these variables. We will check the self-consistency of these Ansätze after we get the results.

We approximate (29) by

$$
\frac{a_{0,1}}{\left|a_{0,2}\right|} e^{-L\left(\frac{1}{a}-1\right)}=a \frac{\sinh \left(Z_{\times} / a\right)}{\cosh Z_{\times}} \approx \tanh Z_{\times} \approx Z_{\times}
$$

This soon leads to the following asymptotic form for the interface potential

$$
V(\ell) / \sqrt{A} \approx-a_{0,2}^{2} e^{-2 L}-2 a_{0,1} a_{0,2} e^{-L(1+1 / a)}-\left(a_{0,1}^{2} / a\right) e^{-2 L / a}+\mathcal{O}\left(e^{-4 L}\right),
$$

where we have no calculational evidence for a possible correction of order $\exp (-4 L)$ but prudently allow for it on general grounds (cf. our remarks following (35)). Note that this possible correction can, depending on the value of $a$, move forward in our expansion and even become the next-to-leading term. In any case, for $1 / 3<a<1$ the first two terms in (40) suffice to obtain a clear prediction for the equilibrium wetting layer thickness $\hat{\ell}$ and the spreading coefficient singularity. The generalization of $(36)$ reads

$$
e^{-\hat{L}\left(\frac{1}{a}-1\right)} \approx \frac{\left|a_{0,2}\right|}{(1+1 / a) a_{0,1}} \propto b
$$

We can now verify that $z_{\times}(\hat{\ell})$ and $z_{\times}(\hat{\ell}) / a$ are indeed bounded below 1 . Note that (41) and (39) imply

$$
\frac{a_{0,1}}{\left|a_{0,2}\right|} e^{-\hat{L}\left(\frac{1}{a}-1\right)} \approx \frac{a}{a+1}=Z_{\times}(\hat{L})
$$

in our (linear) approximation for small $Z_{\times}$. On the other hand, if at this stage we revert back to the hyperbolic functions, we obtain values of $Z_{\times}$that satisfy

$$
\frac{\sinh \left(Z_{\times}(\hat{L}) / a\right)}{\cosh Z_{\times}(\hat{L})}=\frac{1}{a+1},
$$

which is solved rather well by our approximation $(39)$, i.e., $Z_{\times}(\hat{L}) / a \approx 1 /(a+1)<1$. In particular, for $a \rightarrow 0$, $Z_{\times}(\hat{L} / a) \approx 0.8814$ solves $(43)$.

Next, we discuss the surface free energy singularity at wetting. Eliminating $L$ in any of the terms in (40) with use of (41) we find that (38) generalizes to

$$
-S \propto b^{\frac{2}{1-a}}
$$

If we now inspect the results and notational conventions featured in our survey (see Section II.D, final paragraph) we arrive at the conclusion that

$$
2-\alpha_{s}=\frac{1}{1-a}
$$

is consistent with the interpretation that in our model not $b$ but $b^{2}$ is the natural variable to measure the distance to the wetting transition when this transition takes place for $b \rightarrow 0$. Remarkably, for $a<1 / 2$ the wetting transition in our model does not "lock in" to a standard second-order transition with $\alpha_{s}=0$, as in the other models discussed in Section II.D. Instead, the order of the transition decreases continuously from 2 to $3 / 2$, when $a$ is lowered from $1 / 2$ towards $1 / 3$. This is due to the particular form of the exponents of the leading and subleading terms in our interface potential (40), which is somewhat different from that in the other models.

High-precision numerical computations for our model corroborate the conclusions (values of $\alpha_{s}$ and $\beta_{s}$ ) reached on the basis of the analytic interface potential approach and the accompanying approximations we used. What happens, then, for $a<1 / 3$ ? The analytical arguments we presented leave open three possibilities. First, if the amplitude, which we denote by $B$, of the correction term of order $\exp (-4 L)$ to $(40)$ is positive, the order of the transition takes 


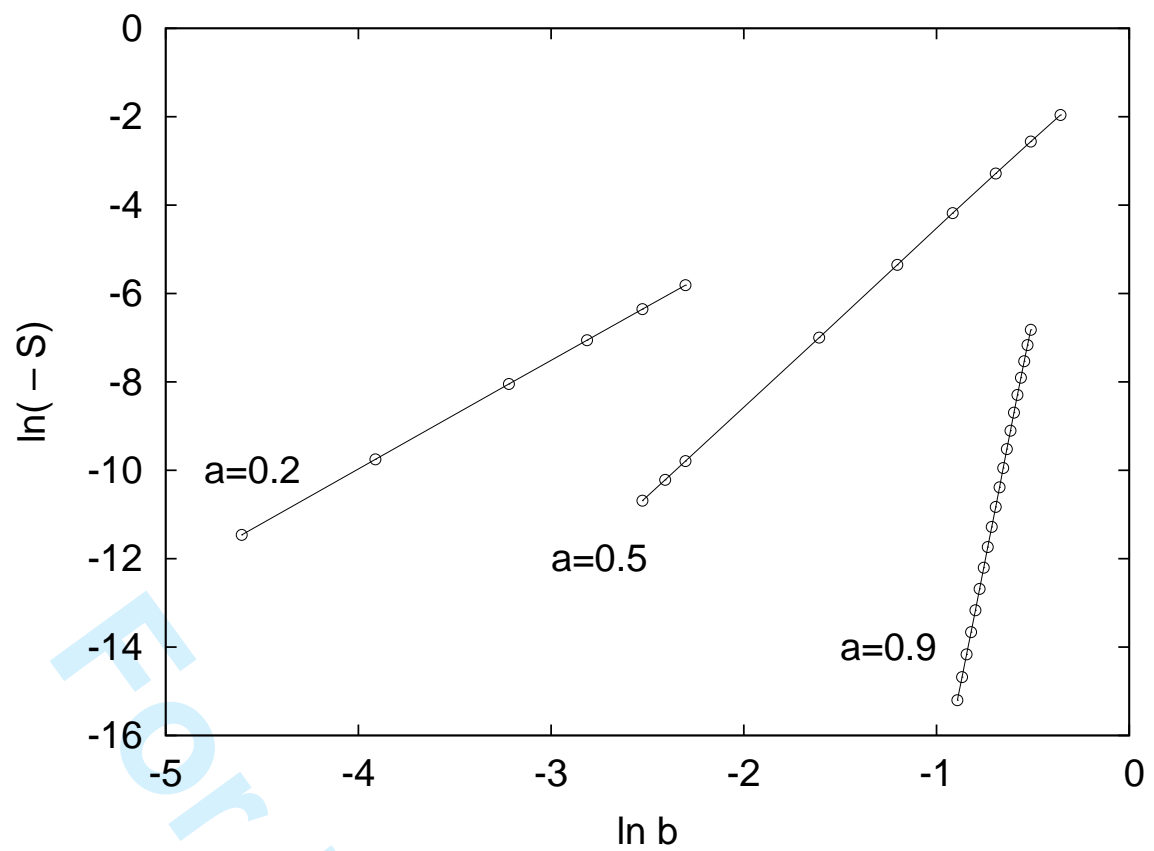

FIG. 6: Equilibrium spreading coefficient $S$ versus wetting parameter $b$, in log-log representation, for selected values of the asymmetry parameter $a$. The slopes, which correspond to twice the order of the wetting transition, according to (44) and (45), are $2.5 \pm 0.1$ for $a=0.2,4 \pm 0.2$ for $a=0.5$ and $20 \pm 1$ for $a=0.9$. The wetting transition takes place in the limit $b \rightarrow 0$.

a universal value for $a \leq 1 / 3$. Secondly, if $B=0$ the transition remains non-universal also below $a=1 / 3$, until a higher-order correction becomes relevant. Thirdly, if $B<0$ it is less obvious what to expect, but a (non-universal) critical wetting transition in the limit $b \rightarrow 0$ is still possible provided $B \rightarrow 0$. Our numerical computations are consistent with the second (or third) scenario. The non-universal critical wetting transition appears to persist, at least down to $a=1 / 5$, at which point we obtain $-S \propto b^{5 / 2}$, in agreement with (44).

Fig.6 and Fig.7 illustrate the results of our numerical computations for selected values of $a$, for the original model defined through (3) and (4). Fig.6 shows the behavior of the spreading coefficient when the critical wetting transition at $b_{w}=0$ is approached for $a=0.2, a=0.5$ and $a=0.9$. The results are in quantitative agreement with the analytic prediction (44) that the order of the transition is, respectively, $2-\alpha_{s}=1.25,2$ and 10 . Fig.7 shows the behavior of the wetting layer thickness approaching the critical wetting transition at $b_{w}=0$, for $a=0.5$, and approaching the second-order critical wetting transition at $b_{w}=b_{w}(1)=0.618 \ldots$ for $a=1$. In both cases the divergence of $\ell$ is logarithmic, in accord with the analytical arguments which we presented for $a<1$ and with general expectations for $a=1$ (see our discussion in Section II.B, in particular result (9)). Incidentally, these computations for the wetting layer thickness, for $a=1$, complement the earlier paper which uncovered the second-order wetting transition point in this model and in which the behavior of the spreading coefficient was discussed [16]).

\section{Infinite-order wetting in the limit $a \uparrow 1$}

The observation that the wetting phase boundary in the global wetting phase diagram (Fig.4) features a vertical segment at $a=1$ has prompted us to study what happens as this segment is approached from the left. Numerical computation reveals that the wetting transition approached in this manner is of very high, possibly infinite, order. Concomitantly, the wetting layer thickness diverges as the first inverse power of the distance to the phase boundary, a much more dramatic increase than the ubiquitous logarithmic growth found under almost all other circumstances in our and other models with short-range forces.

Analytical support for the occurrence of a genuine infinite-order wetting transition is gathered by examining the interface potential (40) under a different angle. Instead of varying $a_{0,2}$, or, equivalently, $b$, towards zero, we keep this parameter constant and vary $a$ towards 1 . Recalling (40) and the result for the equilibrium wetting layer thickness (41), which we rewrite as follows

$$
\hat{\ell} \sqrt{A} \equiv \hat{L} \approx \frac{a}{1-a} \ln \left(\frac{(1+1 / a) a_{0,1}}{\left|a_{0,2}\right|}\right)
$$




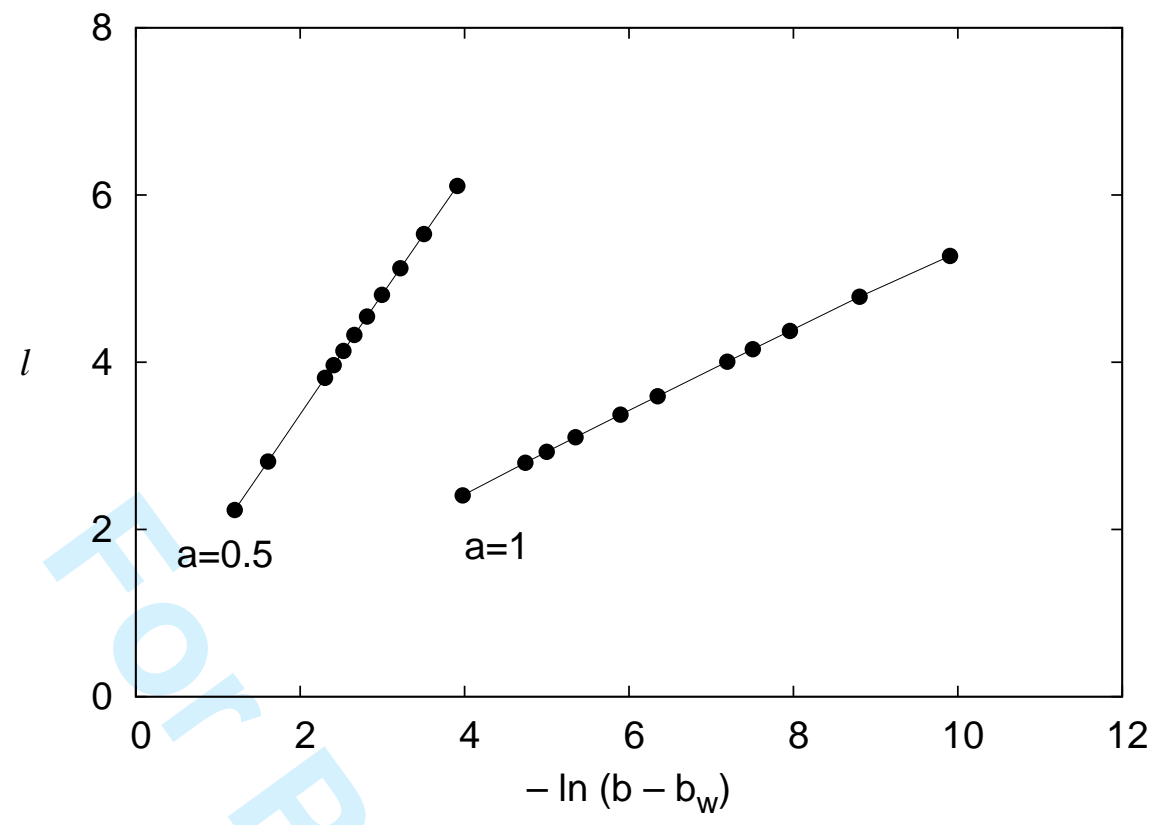

FIG. 7: Equilibrium wetting layer thickness $\ell$ versus wetting parameter $b$, in semi-log representation, for selected values of the asymmetry parameter $a$. For $a=0.5$ the critical wetting transition takes place in the limit $b \rightarrow 0$, while for $a=1$ it takes place at $b=b_{w}(1) \approx 0.681$. In both cases the divergence of $\ell$ is logarithmic.

the algebraic divergence of $\hat{\ell}$ for $a \uparrow 1$ is conspicuous. Likewise, embedding this result in (40) leads to

$$
-S \propto\left(b / b_{w}(1)\right)^{2 a /(1-a)} \propto e^{-C /(1-a)},
$$

with $C>0$, which is possible provided $b<b_{w}(1)=0.681 \ldots$, where $b_{w}(1)$ corresponds to the upper end of the infinite-order transition line in the $(a, b)$-plane at $a=1$. The infinite-order character of the transition (exponential singularity) is thus clearly supported by analytical arguments.

In closing this Section we draw attention to the fact that the variational approach which underlies our analytical results was presented in a different way in our Letter [2]. There, no derivation was presented of the interface potential. In fact, the behavior of the wetting layer thickness was not discussed. The constraint was formulated in terms of the order parameter $\rho_{1}$ rather than in terms of the distance $z$. That approach is equivalent to the one we have employed in this paper.

\section{A SIMPLE(R) MODEL WITH AN INFINITE-ORDER WETTING TRANSITION}

Instead of the 6th-order free energy density function (4) we now consider the 4th-order form

$$
F\left(\rho_{1}, \rho_{2} ; c, b\right)=c \rho_{1}^{2} \rho_{2}^{2}+\left(\rho_{2}-b+b \rho_{1}^{2}\right)^{2}
$$

The bulk phases correspond, as before, to the points $(-1,0),(1,0)$ and $(0, b)$ in the $\left(\rho_{1}, \rho_{2}\right)$-plane. When this free energy density is expanded about the wetting phase, at $\left(\rho_{1}, \rho_{2}\right)=(0, b)$, we obtain the "potential energy"

$$
V\left(\rho_{1}, \rho_{2} ; c, b\right) \equiv-F\left(\rho_{1}, \rho_{2} ; c, b\right) \approx-c b^{2} \rho_{1}^{2}-\left(\rho_{2}-b\right)^{2}
$$

so that we can define the asymmetry parameter $a$ through

$$
\frac{1}{a}=b \sqrt{c}
$$

Chronologically, before we began to explore the properties of the two-parameter model defined through (4), we investigated numerically the model (48) for a fixed value of $c$, e.g., $c=16$. We did this while searching for models 


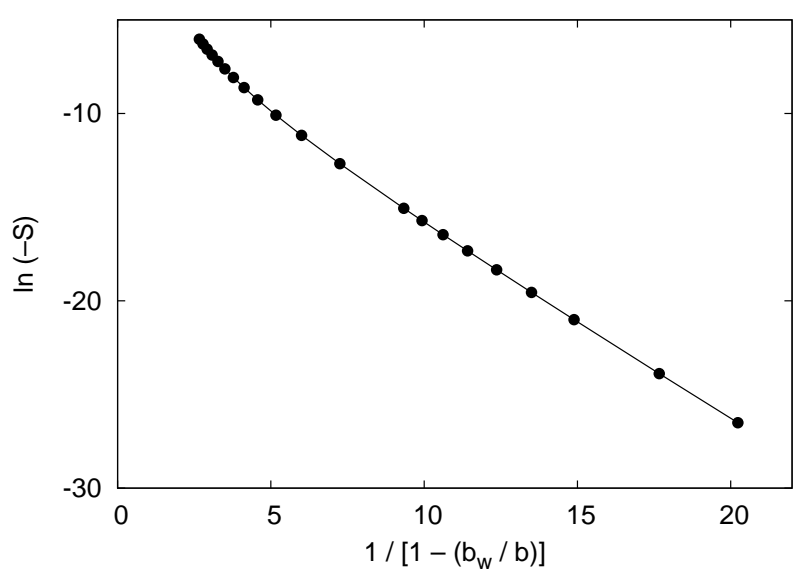

FIG. 8: Equilibrium spreading coefficient $S$ versus wetting parameter $b$, in semi-log representation, for the model defined in (48), with $c=16$ and assuming $b_{w}$ equals the analytically predicted value $1 / 4$. Clearly, the slope converges rapidly to a finite value, supporting the conjectured exponential singularity associated with a wetting transition of infinite order at $b_{w}=1 / 4$.

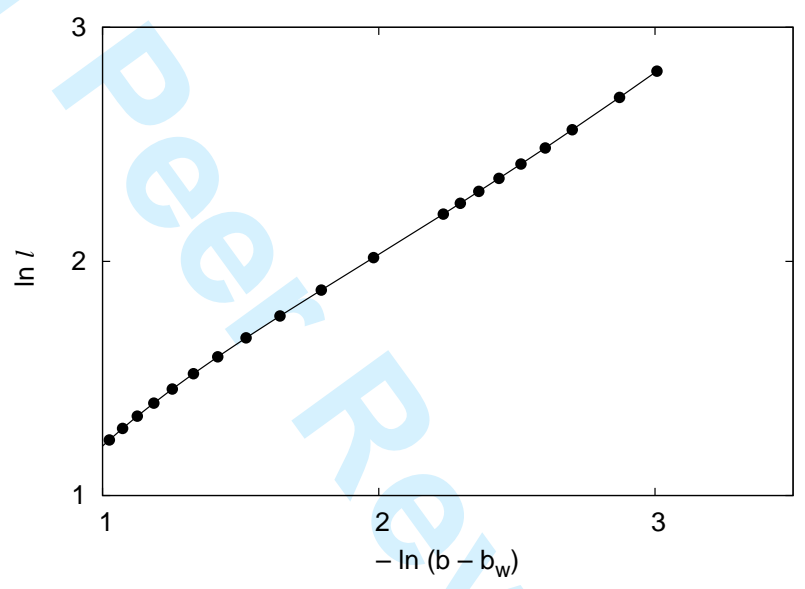

FIG. 9: Equilibrium wetting layer thickness $\ell$ versus wetting parameter $b$, in log-log representation, for the model defined in (48), with $c=16$ and assuming $b_{w}$ equals the analytically predicted value $1 / 4$. Although the data appear to settle along a straight line, the slope still tends to increase slightly as the wetting transition is approached. With our present accuracy we conclude $\beta_{s}=-0.85 \pm 0.2$, with a downward trend. This is not inconsistent with the predicted value $\beta_{s}=-1$.

with a critical wetting transition and with an $F$-polynomial of order lower than six, knowing that for a 6 th-order $F$ a critical wetting transition was already established [16]. Surprisingly, numerical analysis of this quartic-polynomial model with one parameter, $b$, suggested a wetting transition of "very high and possibly infinite" order at $b_{w} \approx 1 / 4$.

Assuming that the character of the singularity of the surface free energy at wetting is governed by the properties of the model in the vicinity of the $\beta$ phase point in the $\left(\rho_{1}, \rho_{2}\right)$-plane, it suffices to insert $c=16$ in $(50)$ in order to understand that this model "lies on" a hyperbola in the $(a, b)$ wetting phase diagram (Fig.4), which crosses the wetting phase boundary in the segment of infinite-order transitions located at $a=1$, in the point $\left(1, b_{w}\right)$ with $b_{w}=1 / 4$. More generally, this argument predicts an infinite-order transition for the two-parameter model (48) provided $c$ is chosen large enough so that $b_{w}=1 / \sqrt{c}<b_{w}(1)$, where $b_{w}(1)$ is a model-dependent threshold.

The prediction of an infinite-order transition is in accord with the numerical computations for this model, illustrated in Fig. 8 and Fig.9. Fig.8 shows the behavior of the spreading coefficient when the critical wetting transition at $b_{w}=1 / 4$ is approached for $c=16$. The results are in quantitative agreement with the analytic prediction of an exponential singularity (47), implying that the order of the transition is infinity. Fig.9 shows the behavior of the wetting layer thickness approaching the infinite-order wetting transition. The divergence of $\ell$ appears to be algebraic, in agreement with the analytical prediction (46), which can be written in the form

$$
\hat{\ell} \propto \frac{a}{1-a} \propto \frac{b_{w}}{b-b_{w}}
$$


in view of (50). However, the slope of the line formed by the data in Fig.9 has apparently not yet converged. With our present accuracy we cannot yet conclusively confirm the value -1 predicted by (51).

\section{CONCLUSIONS AND OUTLOOK}

We close this paper with a concise recapitulation and some speculations. The phase diagram (Fig.4) teaches us that fairly basic choices of density-functional models for three-phase equilibria possess an astonishingly rich variety of wetting phase transitions. The infinite-order transitions, to which we draw special attention, and which were almost not recognized until very recently, appear to be fairly ubiquitous! Indeed, while they seem to emerge for the first time, within mean-field theory, in the two-parameter model studied in our Letter [2], we conjecture that a segment of infinite-order wetting also occurs in the global wetting phase diagram of two previously studied multi-component order parameter models [3, 20]. From a theoretical point of view, a segment of infinite-order transitions (Fig.4) appears to be a new alternative to known scenarios for connecting first-order to critical wetting, such as the familiar tricritical point [31] and the less familiar critical endpoint scenario [21, 32].

Moreover, we suggest that the ferromagnet with cubic anisotropy might well be the most obvious experimental system in which to look for the infinite-order transition. Since in general the (cubic) magnetic anisotropy, and consequently the parameter $a$, is temperature dependent [33], varying the temperature implies following an oblique path in the global temperature-anisotropy phase diagram (Fig.4). Here we assume that $b$ is a temperature-like or at least temperature-dependent field, which is explicitly realized in (13), where $b \propto \sqrt{-t}$. Therefore, the infinite-order wetting transition ought to be accessible in this system, no less than the wetting transitions of finite order and the experiment can, in principle, be performed on a single sample by varying $T$. Further, note that the path traced by varying the control parameter in the simpler model studied in Section IV, corresponds to an oblique path in the $(a, b)$-plane.

How do thermal fluctuations, not included in our mean-field treatment, affect our results, knowing that the upper critical dimension for wetting and short-range forces is $d=3[14,26]$ ? We follow the reasoning advocated in [3] and acknowledge that, when the wetting transition takes place sufficiently far from bulk (multi-)criticality, the results are not significantly altered. Otherwise, exponent renormalization is in order, and the results presented in [19] can provide a guideline, to a large extent also for our present model. In any case, this is an open problem outside the scope of our paper.

In this paper we have gone beyond what was presented in our Letter [2] in several respects. We have, inter alia, derived new results for the behavior of the wetting layer thickness $\ell$ upon approach of an infinite-order wetting transition, in particular regarding the algebraic divergence $\left(\beta_{s}=-1\right)$, and we have outlined how analytic approximations can be obtained and used to anticipate, or explain, the findings of accurate numerical analysis of the full density-functional model. It is peculiar to the infinite-order transition that analytic arguments are indispensable to complement "exact" numerical computations, because it is subtle to distinguish a transition of very high but still finite order from one of infinite order.

\section{ACKNOWLEDGEMENT}

We wish to express our deep appreciation and admiration of Bob Evans' pioneering works in statistical and soft condensed matter physics which have been a continuous source of inspiration for us and countless others. We also gratefully acknowledge Bob's generous critical opinion and plentiful spiritual remarks on many of our own works. It is with pleasure and excitement that we dedicate this contribution to him on the occasion of his 65 th birthday, since wetting phenomena have enjoyed his special attention since the late 70's when they became irrevocably entangled with phase transitions and critical phenomena.

[1] For a recent review, see D. Bonn, J. Eggers, J.O. Indekeu, J. Meunier and E. Rolley, Rev. Mod. Phys. 81, 739 (2009).

[2] K. Koga, J.O. Indekeu and B. Widom, Phys. Rev. Lett. 104, 036101 (2010).

[3] E.H. Hauge, Phys. Rev. B33, 3322 (1986).

[4] J.S. Rowlinson and B. Widom, "Molecular theory of capillarity" (Dover, New York, 2002).

[5] R. Evans, Adv. Phys. 28, 143 (1979); R. Evans, Chapter 3, pp. 85-175, in "Fundamentals of Inhomogeneous Fluids" (ed. D.Henderson; Dekker, 1992); and R. Evans, in "Liquids at Interfaces, Proceedings of the Les Houches Summer School of Theoretical Physics", Les Houches, 1988 (eds. J. Charvolin, J. F. Joanny, and J. Zinn-Justin North Holland, Amsterdam, 1990). 
[6] R. Roth, R. Evans, A. Lang and G. Kahl, J. Phys. Cond. Matter 14, 12063 (2002).

[7] P. Hopkins, A. J. Archer and R. Evans, J. Chem. Phys. 129, 214709 (2008); P. Tarazona and R. Evans, Mol. Phys. 48, 799 (1983).

[8] M.R. Moldover and J.W. Cahn, Science 207, 1073 (1980).

[9] D. Bonn, H. Kellay and G. Wegdam, Phys. Rev. Lett. 69, 1975 (1992).

[10] We tacitly ignore the fact that in this system, at the elevation of the vapor-liquid interface, the adsorbate is slightly away from two-phase coexistence. If we were to take this into account, we ought to describe the observed phenomena in terms of a first-order prewetting transition. This does, however, not affect its discontinuous character essential to our present discussion.

[11] I. Szleifer and B. Widom, Mol. Phys. 75, 925 (1992).

[12] K. Koga and B. Widom, J. Chem. Phys. 127, 064704 (2007).

[13] D. B. Abraham, Phys. Rev. Lett. 441165 (1980).

[14] For a review, see M.E. Fisher, J. Chem. Soc. Faraday Trans. II 82, 1569 (1986).

[15] D. Ross, D. Bonn and J. Meunier, Nature 400, 737 (1999).

[16] K. Koga and B. Widom, J. Chem. Phys. 128, 114716 (2008).

[17] K. Ragil, J. Meunier, D. Broseta, J. Indekeu and D. Bonn, Phys. Rev. Lett. 77, 1532 (1996).

[18] N.R. Bernardino, A.O. Parry, C. Rascon, and J.M. Romero-Enrique, J. Phys. Condens. Matter 21, 465105 (2009), and references therein.

[19] T. Aukrust and E.H. Hauge, Phys. Rev. Lett. 54, 1814 (1985).

[20] C.J. Walden, B.L. Györffy and A.O. Parry, Phys. Rev. B 42, 798 (1990).

[21] J.O. Indekeu and J.M.J. van Leeuwen, Phys. Rev. Lett. 75, 1618 (1995); Physica C 251, 290 (1995).

[22] Incidentally, the special case $a=1$, or $\lambda=3$, does not represent a "tricritical point" as suggested in [20], but rather a (simple) critical point, with, indeed, the universal exponent of second-order wetting [20]. This critical point is, in our view, the terminus of a line of infinite-order wetting transitions, located at $\lambda=3$. These infinite-order transitions can take place upon varying $\lambda$ towards the value 3 from above (to be compared with the vertical segment at $a=1$ in Fig.4). At the same time, this critical point can be the endpoint of a line of first-order wetting transitions which extends towards $a>1$, or $\lambda<3$ (cf. Fig. 4).

[23] V.F. Kozhevnikov, M.J. Van Bael, P.K. Sahoo, K. Temst, C. Van Haesendonck, A. Vantomme and J.O. Indekeu, New J. Phys. 9, 75 (2007).

[24] J.M.J. van Leeuwen and E.H. Hauge, J. Stat. Phys. 87, 1335 (1997).

[25] W. Speth, PhD thesis (München 1986). A summary of Speth's contribution is given in [26].

[26] S. Dietrich, in "Phase transitions and critical phenomena", vol.12, eds. C. Domb and J.L Lebowitz (Academic, London, 1988) p.1.

[27] F. Clarysse and J.O. Indekeu, Physica A 521, 70 (1998).

[28] R. Lipowsky and Th.M. Nieuwenhuizen, J. Phys. A 21, L89 (1988).

[29] The existence of this intersection can be demonstrated analytically as follows. Without loss of generality, fix the asymmetry parameter to $a=1 / 2$. The approximate non-wet trajectory is then given by $\rho_{1}(Z)=2 a_{1} \sinh 2 Z$ and $\rho_{2}(Z)=b-4 a_{1} \cosh Z$. At position $Z=\tilde{Z}$ given by $\cosh \tilde{Z}=b /\left(4 a_{1}\right)$ it crosses the axis $\rho_{2}=0$, at $\rho_{1}(\tilde{Z})=\sqrt{1-\left(4 a_{1} / b\right)^{2}} b^{2} /\left(4 a_{1}\right)$. If we choose $a_{1}<b^{2} /\left(4 \sqrt{1+b^{2}}\right)$ we obtain $\rho_{1}(\tilde{Z})>1$. This is a sufficient condition for the existence of an intersection of the approximate non-wet trajectory and the exact wet trajectory in the $\left(\rho_{1}, \rho_{2}\right)$-plane.

[30] Strictly speaking, at $z=z_{\times}$, the harmonic approximations to the non-wet and wet trajectories near $\beta$ intersect. In Fig.5 only the exact wet trajectory is shown, but the distinction is negligible on the scale of the figure.

[31] H. Nakanishi and M.E. Fisher, Phys. Rev. Lett. 49, 1565 (1982).

[32] S. Rafaï, D. Bonn, E. Bertrand, J. Meunier, V.C. Weiss and J.O. Indekeu, Phys. Rev. Lett. 92, 245701 (2004).

[33] P. Asselin, R.L.F. Evans, J. Barker, R.W. Chantrell, R. Yanes, O. Chubykalo-Fesenko, D. Hinzke and U. Nowak, Phys. Rev. B 82, 054415 (2010); for other possibilities (than changing the temperature) of manipulating magnetic anisotropy experimentally, see the review M. T. Johnson, P. J. H. Bloemen, F. J. A. den Broeder and J. J. de Vries, Rep. Prog. Phys. 59, 1409 (1996). 
(a)

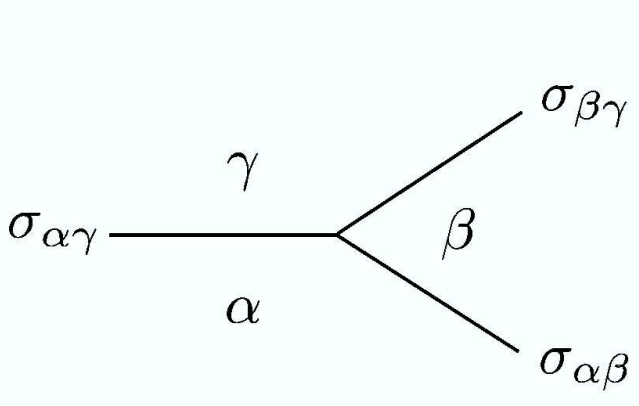

(b)

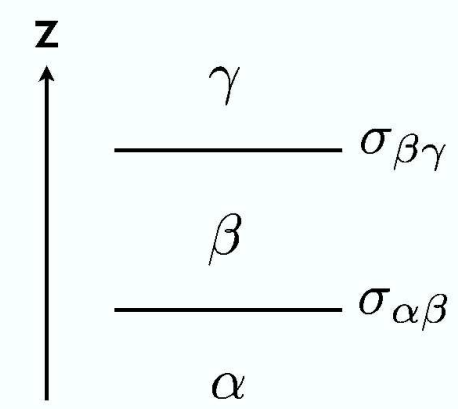

$282 \times 282 \mathrm{~mm}(600 \times 600 \mathrm{DPI})$

URL: http://mc.manuscriptcentral.com/tandf/tmph 


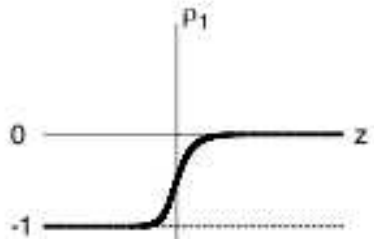

(a)

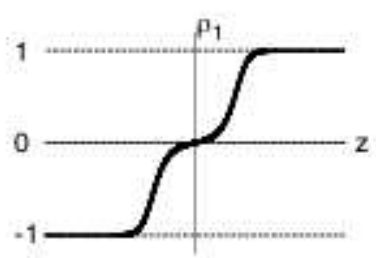

(c)

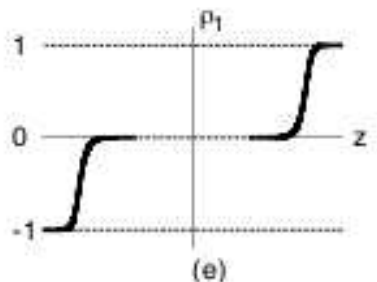

(e)

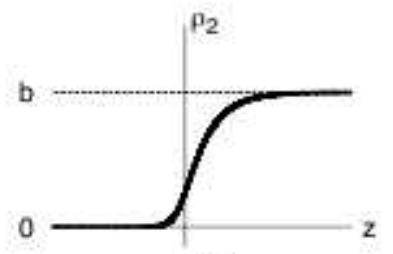

(b)

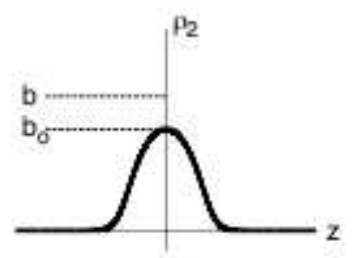

(d)

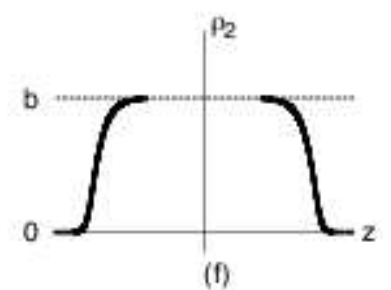

(f)

$$
92 \times 101 \mathrm{~mm}(100 \times 100 \mathrm{DPI})
$$




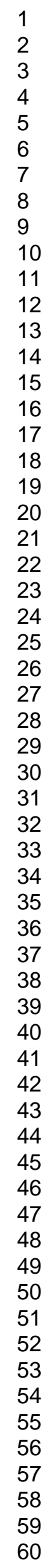

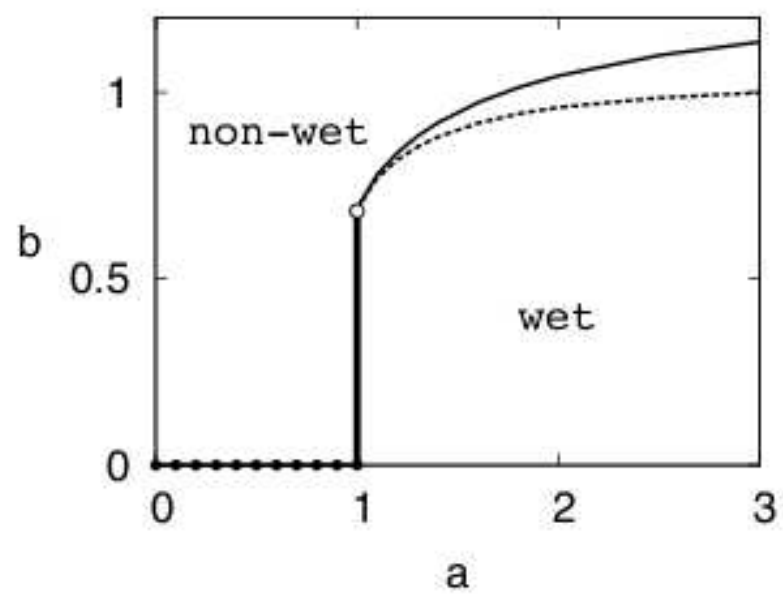

$88 \times 61 \mathrm{~mm}(100 \times 100 \mathrm{DPI})$

URL: http://mc.manuscriptcentral.com/tandf/tmph 